\title{
Overexpression of the chimeric plasmin-resistant VEGF165/VEGF183 (132-158) protein in murine breast cancer induces distinct vascular patterning adjacent to tumors and retarded tumor growth
}

\author{
HUI-YONG ZHANG ${ }^{1}$, BING-LIN FAN ${ }^{2}$, XIN-SHENG WU ${ }^{3}$, LING-MIN MU ${ }^{2}$, \\ WEN-FENG WANG ${ }^{1}$ and WU-LING $\mathrm{ZHU}^{2}$ \\ ${ }^{1}$ College of Life Science and Biotechnology; ${ }^{2}$ School of Basic Medical Sciences, Xinxiang Medical University; \\ ${ }^{3}$ Department of Vasculocardiology, Xinxiang 371 Central Hospital, Xinxiang, Henan 453003, P.R. China
}

Received January 14, 2014; Accepted August 19, 2014

DOI: $10.3892 / \mathrm{mmr} .2014 .2866$

\begin{abstract}
A chimeric plasmin-resistant vascular endothelial growth factor (VEGF)165/VEGF192 (132-158) protein, named as VEGF192 (according to the nomenclature of VEGF), designed by a previous study, was demonstrated to have an enhanced affinity for the extracellular matrix (ECM) amongst other bioactivities. However, it is now accepted that mutant VEGFs frequently demonstrate different angiogenic activities and produce different vascular patterning from the parental molecule. The present study hypothesized that VEGF192, due to its enhanced binding affinity to the ECM, would exhibit a different angiogenic activity and produce a different vascular patterning compared to those of VEGF165.Murine breast cancerEMT-6 cells were manipulated to stably overexpress VEGF165 or VEGF192. These cells were then inoculated intradermally into BALB/c mice in order to monitor the formation of vascular patterning in skin proximal to tumors. In vivo angiogenesis experiments revealed that overexpression of VEGF192 in murine breast cancer cells resulted in irregular, disorganized and dense vascular patterning as well as induced a significant inhibition of tumor growth compared with that of VEGF165. In addition, allograft tumor immunochemical assays of VEGF192-overexpressing tumors demonstrated significantly lower vascular densities than those of VEGF165-overexpressing tumors; however, VEGF192 tumors had a significantly enlarged vascular caliber. Conversely, cell wound healing experiments revealed that VEGF192-overexpressing EMT-6 cells had significantly decreased migration rates compared with those of VEGF165-overexpressing EMT-6 cells. In conclusion, the results of the present study supported the hypothesis that the
\end{abstract}

Correspondence to: Professor Wu-Ling Zhu, School of Basic Medical Sciences, Xinxiang Medical University, 61 Jinsui Road, Xinxiang, Henan 453003, P.R. China

E-mail: Zhuwuling1@163.com

Key words: vascular endothelial growth factor, vascular patterning, angiogenesis, breast cancer altered ECM affinity of VEGF induced structural alterations to vasculature. In addition, these results provided a novel insight into VEGF design and indirect evidence for the function of exon 8 in VEGF.

\section{Introduction}

Vascular endothelial growth factor A (VEGF) is a clinically significant angiogenic agent used for its potent function in angiogenesis in the treatment of numerous ischemic diseases, including coronary heart disease as well as chronic wound and diabetic lower limb ischemias $(1,2)$. However, VEGF has restricted clinical application due to its short half-life and the potential side effects of an overdose, which include edema, inflammation, hemorrhage, hypotension and hemangioma (3-5). Therefore, the identification or design of a novel endothelial cell-specific proangiogenic factor with fewer side effects is required.

A previous study generated a chimeric VEGF called VEGF192 (according to the nomenclature of VEGF) from the fusion of the VEGF183 (132-158, KDRARQENKSVRGK GKGQKRKRKKSR) peptide to the COOH-terminus of the plasmin-resistant VEGF165 (6). The peptide corresponded to the amino acid sequence 132-158 of VEGF183 following the cleavage signal sequence and contained the plasmin and matrix metalloproteinase (MMP) cleavage sites, as well as extracellular matrix (ECM) binding sequences (7). The study reported that the VEGF192 chimeric proteins demonstrated a stronger affinity for the ECM in a hypoxic environment, enabling them to be cleaved by MMPs and plasmin, and therefore retain their mitogenic activity for endothelial cells. In addition, it was reported that VEGF192 reduced vascular permeability and had an increased half-life in vivo compared to that of VEGF165 (6). Furthermore, Tammela et al reported that modifying VEGF isoforms through the addition of novel domains may result in 'designer' VEGFs with different bioactivities from those of the parent molecules $(8,9)$.

In the majority of cases mutant or cleaved VEGFs demonstrate altered angiogenic activities and vascular patterning 
than those of the parental molecules (9). Keskitalo et al (10) generated a chimeric VEGF/VEGF-C silk domain fusion protein, which resulted in a chimeric VEGF-CAC protein that significantly enhanced capillary formation compared to that of the VEGF parent molecules (10). In addition, Tammela et al (8) constructed a chimeric protein composed of VEGF-C/VEGF heparin-binding domain fusion proteins, which was reported to stimulate distinct structural changes to lymphatic vessels. Zheng et al (11) designed a chimeric VEGF-E (NZ7)/placental growth factor fusion protein that was shown to be capable of promoting angiogenesis via VEGFR-2 without significant enhancement of vascular permeability and inflammation. Lee et al (12) suggested that matrix-bound or non-tethered VEGF provided different signaling and vascular patterning activities compared to those of the parent molecules. The present study hypothesized that due to the enhanced binding affinity of VEGF192 to ECM, it may induce differential vascular patterning compared with that of VEGF165.

The aim of the present study was to explore the biological effects of VEGF192 in vivo. Murine breast cancer EMT-6 cells were manipulated to stably overexpress VEGF165 or VEGF192. These cells were then inoculated intradermally into BALB/c mice in order to monitor the formation of vascular patterning in the skin proximal to tumors.

\section{Materials and methods}

Experimental procedures were approved by and all animal experiments were conducted in accordance with the current regulations and standards of the Animal Ethics Study Committee of Xinxiang Medical University (Xinxiang, China).

Cell lines and animals. The EMT- 6 murine breast cancer cell line was purchased from the American Type Culture Collection (Manassas, VA, USA) and grown in Dulbecco's modified Eagle's medium (DMEM; HyClone ${ }^{\text {TM}}$; Waltham, MA, USA) supplemented with $10 \%$ fetal bovine serum (FBS; Gibco-BRL, Carlsbad, CA, USA), $100 \mathrm{U} / \mathrm{ml}$ penicillin and $100 \mu \mathrm{g} / \mathrm{ml}$ streptomycin (Sangon Biotech, Shanghai, China). Female BALB/c mice (Experimental Animal Center of Henan Province, Zhengzhou, China), aged six to eight weeks, were used for all experiments. Female BALB/c mice were raised in a specific pathogen-free environment throughout the experiment with a constant room temperature of $20-25^{\circ} \mathrm{C}$ and constant humidity of $35-40 \%$ with a 12 -h light-dark cycle and were given free access to food and water.

Constructs. The full-length recombinant human VEGF165 was provided by Dr Liu Jingjing (minigene lab of China Pharmaceutical University, Nanjing, China). The VEGF165 mutant resistant to plasmin proteolysis was generated as described by Lauer et al (13). Construction of VEGF192 was performed using polymerase chain reaction (PCR)-mediated mutagenesis as previously described (7).

The novel VEGF192 and VEGF165 constructs, harboring their native kozak sequence, were inserted into the mammalian expression vector pcDNA3.1plus (Invitrogen Life Technologies, Carlsbad, CA, USA) and further characterized using DNA sequencing (GENEWIZ, Inc., Suzhou, China).
Stable gene transfection. Endotoxin-free plasmids pcDNA3.1plus-VEGF165, pcDNA3.1plus-VEGF192 and the control vector pcDNA3.1plus were extracted using the E.Z.N.A. ${ }^{\mathrm{TM}}$ endo-free plasmid midi kit (Omega Bio-Tek, Norcross, GA, USA) according to the manufacturer's instructions. Stable transfections of EMT-6 cells were performed using the Lipofectamine ${ }^{\mathrm{TM}} 2000$ reagent according to manufacturer's instructions (Invitrogen Life Technologies). Parental cells were also transfected using the pcDNA 3.1plus vector (control plasmid). Transfected cells were incubated in complete growth medium containing $600 \mu \mathrm{g} / \mathrm{ml}$ G418 antibiotics (Sangon Biotech) for 1-2 weeks.

Stably transfected individual clones were generated using serial dilutions of G418. G418-resistant clones to be screened in complete medium plus $600 \mu \mathrm{g} / \mathrm{ml} \mathrm{G} 418$, and then stable transfected individual clones were generated by limited dilutions maintained in complete growth medium containing $300 \mu \mathrm{g} / \mathrm{ml}$ of $\mathrm{G} 418$.

ELISA screening test for stable clones overexpressing VEGF isoforms. Levels of human VEGF were quantified in conditioned medium, prepared as follows: Cells were plated in triplicate at a density of $2.5 \times 10^{5}$ cells per well and incubated overnight in 24-well plates. Cells were then incubated for $48 \mathrm{~h}$ in fresh serum-free medium containing $100 \mu \mathrm{g} / \mathrm{ml}$ heparin (Sangon Biotech) in order to release cell-associated VEGF. Conditioned medium was then collected and cellular debris removed using centrifugation at $10,000 \mathrm{xg}$ for $5 \mathrm{~min}$ at $40^{\circ} \mathrm{C}$. A Human VEGF ELISA kit (Wuhan Boster Biological Technology, Ltd., Wuhan, China) was used to measure human VEGF protein levels in conditioned medium according to the manufacturer's instructions. Each sample was measured in duplicate.

$R N A$ extraction and reverse transcription quantitative $P C R$ (RT-semi-qPCR). Confluent EMT-6 cells were cultured in DMEM-10\% FBS. RNA was isolated using TRIzol ${ }^{\circledR}$ reagent according to the manufacturer's instructions (Sangon Biotech). Reverse transcription of $1 \mu \mathrm{g}$ total RNA was performed using 200 U Superscript II RNase H reverse transcriptase with oligo deoxy-thymine (dT; Takara Bio, Inc., Dalian, China).

In order to further semi-quantify the human VEGF gene in the screened clones, RT-semi-qPCR was performed using the following primers: Human VEGF forward (NM_001171622.1), 5'-CTTGCTGCTCTACCTCCACC-3' and VEGF reverse, 5'-GCCTCGGCTTGTCACATCT-3'; GAPDH forward, 5'-AGCGAGACCCCACTAACA-3' and GAPDH reverse, 5'-ATGAGCCCTTCCACAATG-3'. GAPDH was used as the control.

The complementary DNA (cDNA) samples were mixed with the above-mentioned primers and amplified using the following cycling profile: $95^{\circ} \mathrm{C}$ for $5 \mathrm{~min}, 26$ cycles at $95^{\circ} \mathrm{C}$ for $15 \mathrm{sec}$, then either $57^{\circ} \mathrm{C}$ for $30 \mathrm{sec}$ (VEGF) or $55^{\circ} \mathrm{C}$ for $30 \mathrm{sec}(\mathrm{GAPDH})$, followed by $72^{\circ} \mathrm{C}$ for $30 \mathrm{sec}$. The PCR products were visualized using electrophoresis with $1.5 \%$ agarose containing $0.5 \mu \mathrm{g} / \mathrm{ml}$ ethidium bromide solution.

In vivo assay for tumor-adjacent angiogenesis. A total of 24 eight-week-old female BALB/c mice were divided randomly into three groups $(n=8)$, anesthetized by intraperitoneal injection of $3.6 \%$ chloral hydrate solution $(0.2 \mathrm{ml})$ (Sangon Biotech), then 
A

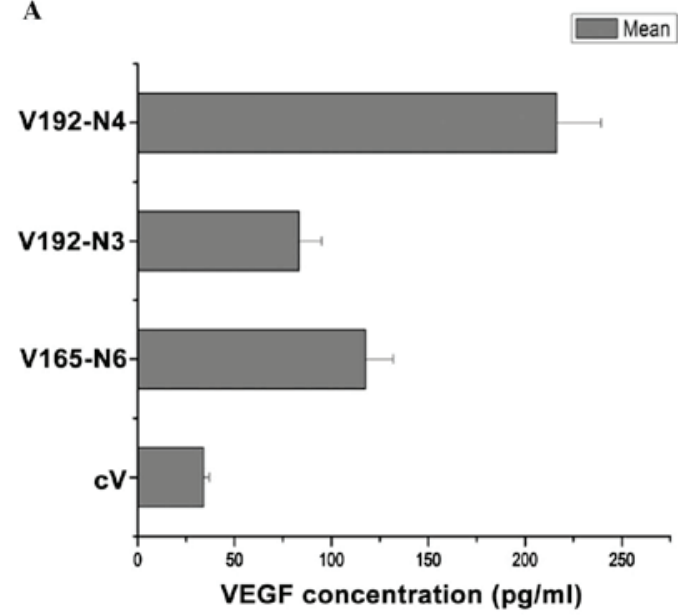

B

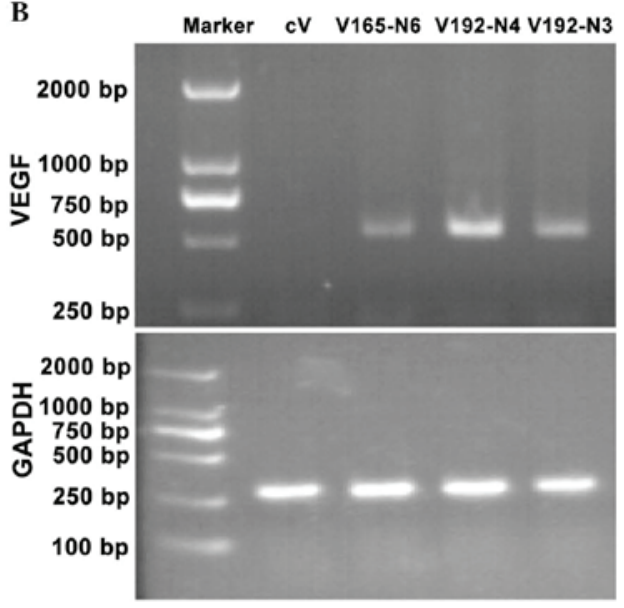

Figure 1. Analysis of VEGF expression in transfected EMT-6 clones. EMT-6 clones were transfected with pcDNA3.1plus vectors expressing three different VEGF isoforms and a control vector. Cells were then treated with heparin $(100 \mu \mathrm{g} / \mathrm{ml})$ and incubated for $48 \mathrm{~h}$. Proteins from the conditioned medium were analyzed using: (A) A human VEGF ELISA kit; and (B) VEGF reverse transcription quantitative polyermase chain reaction with GAPDH as the complementary DNA loading control and visualized using ethidium bromide and ultraviolet transillumination. Data are expressed as the mean \pm standard deviation for three independent experiments performed in duplicate. VEGF, vascular endothelial growth factor A; V165-N6, EMT-6 cells transfected with pcDNA3.1plus-VEGF165; V192-N4 and V192-N3, EMT-6 cells transfected with pcDNA3.1plus-VEGF192; cV, control group of EMT-6 cells transfected with pcDNA3.1plus.

shaved on both flanks. Each group was injected intradermally with a single cell suspension $\left(2.5 \times 10^{5}\right.$ cells in $0.1 \mathrm{ml}$ DMEM) of EMT-6 cell clones overexpressing VEGF192 (V192-N3), EMT-6 cell clones overexpressing VEGF165 (V165-N6) or the parental cells which carried the pcDNA3.1plus vector $(\mathrm{cV})$. Each mouse received two injections of tumor cells on each flank. When the tumors reached similar volumes $\left[28 \pm 3 \mathrm{~mm}^{3}\right.$, based on the formula: Tumor volume $\left(\mathrm{mm}^{3}\right)=$ length $\mathrm{x}$ width $\left.{ }^{2} \mathrm{x} 0.52\right]$, the mice were sacrificed. The inner surface of their abdominal wall skins, which covered the implant sites, was removed and spread onto filter paper. Images of the newly formed blood vessels growing into the injected tumor area were captured using a Canon SX50 HS (Zhuhai, China) camera and were used for analysis.

In vivo tumorigenesis. A total of 24 eight-week-old female $\mathrm{BALB} / \mathrm{c}$ mice were divided randomly into three groups $(\mathrm{n}=8)$. Then $100 \mu \mathrm{l}$ single cell suspension $\left(1 \times 10^{7}\right.$ cells $/ \mathrm{ml}$ in RPMI 1640 medium; HyClone, Beijing, China), of either V192-N3, V192-N4 or V165-N6 EMT-6 cells as well as cV was injected subcutaneously into the flank of mice in the respective groups. N4 is a clone which has a capacity to express more VEGF protein ( $200 \mathrm{pg} / \mathrm{ml})$ than that of clone N3 ( 100pg/ml VEGF), which was used to determine the effect of the VEGF192 expression level on the proliferation of EMT-6. When tumors became palpable, tumor sizes were measured every two days using calipers. Tumor volumes were calculated using the following formula: Tumor volume $\left(\mathrm{cm}^{3}\right)=$ length $\mathrm{x}$ width $\mathrm{x} 0.52$.

Histological and immunohistochemical analysis. Intradermal tumors (6-8 mm diameter; $\sim 28 \mathrm{~mm}^{3}$ ) were fixed with $4 \%$ paraformaldehyde (Zhongshan Bio-tech, Co., Ltd., Beijing, China), dehydrated and paraffin-embedded, then cut into $5-\mu \mathrm{m}$ sections. Sections were stained with hematoxylin and eosin (H.E.) (Zhongshan Bio-tech, Co., Ltd.) according to the manufacturer's instructions. H.E. sections were examined by tumor histologist, Professor Su Ning from the School of Medicine of Southeast University (Nanjing, China). For immunohistochemical analysis, the sections were deparaffinized and rehydrated into phosphate-buffered saline. Antigen retrieval was performed using citrate buffer ( $\mathrm{pH} 6.5$ ) for $30 \mathrm{~min}$ at $92-98^{\circ} \mathrm{C}$. The sections were stained in order to identify endothelial cells using the following antibodies: Rabbit anti-mouse CD31 monoclonal antibody (Sino Biological, Inc., Beijing, China); followed by goat anti-rabbit immunoglobulin $\mathrm{G}$ antibody (Zhongshan Bio-tech, Co., Ltd.). Antibody binding was visualized using 3,3'-diaminobenzidine (Zhongshan Bio-tech, Co., Ltd.) and signal amplification was achieved via the avidin-biotin complex (Zhongshan Bio-tech, Co., Ltd.). Staining was then quantified according to the Chalkley method (14).

Measurement of microvessel density. Tumor vascularization was assessed using the Chalkley method. Areas of highest vascular density ('hot spots') were identified at low magnification in the whole sections (magnification, x100). Magnification was then increased (x200) and a point-counting grid was applied to each hot spot, a minimum of three hot spots were counted in each representative tumor zone. The most representative tumor zones were studied in each case. Results of vessel density were expressed as the mean \pm standard deviation (SD) of vessel number per $\mathrm{mm}^{2}$.

Wound healing. Confluent cell cultures were grown on 24-well plates in DMEM-10\% FBS. Wounds were generated with the tip of a micropipette $(\sim 100 \mu \mathrm{m})$, and cells were maintained in serum-free DMEM for $48 \mathrm{~h}$. Following $24 \mathrm{~h}$, cells were fixed with $4 \%$ paraformaldehyde and stained with crystal violet (Sangon Biotech). Images of three fields of vision were captured and analyzed for each well at x100 magnification. Each experiment was performed in triplicate.

Statistical analysis. Data are presented as the mean \pm SD. One-way analysis of variance was performed to test for differences between groups. Individual group comparisons were performed using the unpaired Tukey's post-hoc test. $\mathrm{P}<0.05$ 


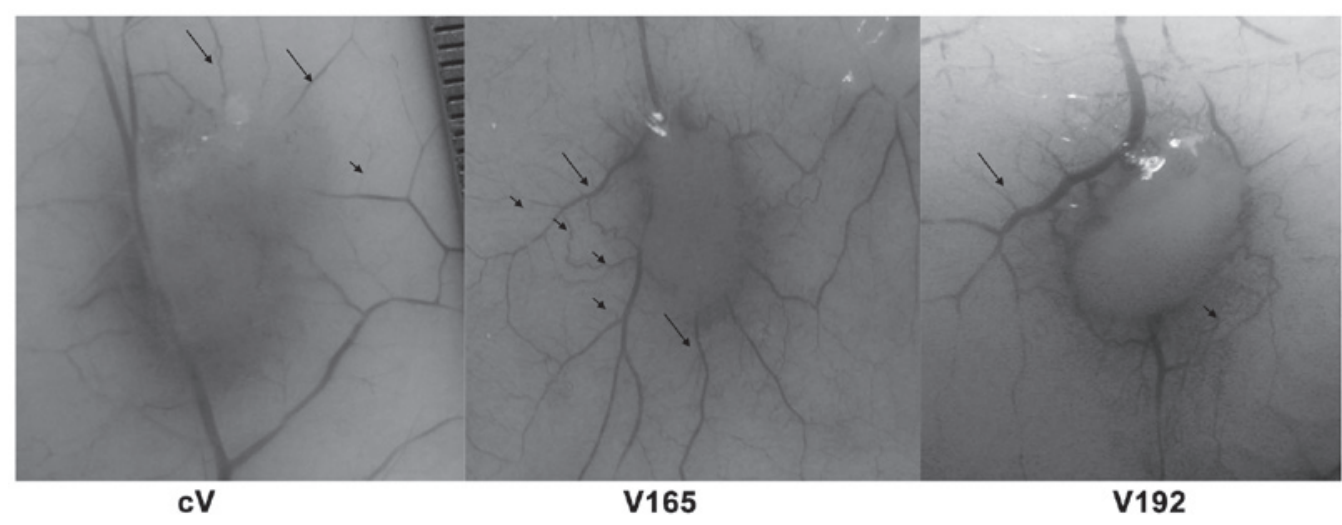

Figure 2. Effects of VEGF165 and VEGF192 on vessels adjacent to tumors. BALB/c mice were administered intradermal injections of V192-N3, V165-N6, and $\mathrm{cV}$, respectively. When the tumors reached a similar volume $\left(28 \pm 3 \mathrm{~mm}^{3}\right)$, mice were sacrificed and images were captured of the newly formed blood vessels growing into the area of injected tumor cells. The macroscopic appearance of tumor and tumor-induced vessel patterns in the skin are shown; long arrows indicate secondary level vessels, and short arrows indicate teritary level vessels. VEGF, vascular endothelial growth factor A; V165, EMT-6 cells overexpressing VEGF165; V192, EMT-6 cells overexpressing VEGF192; cV, control vector group.

Table I. Average number and length of the secondary and tertiary level vessels.

\begin{tabular}{lrrc}
\hline Variable & $\mathrm{cV}$ & $\mathrm{V} 165$ & $\mathrm{~V} 192$ \\
\hline Number of secondary vessels (n) & $8 \pm 2$ & $7 \pm 2$ & $16 \pm 3$ \\
Number of tertiary level vessels (n) & $16 \pm 4$ & $45 \pm 6$ & $37 \pm 6$ \\
Length of secondary vessels (mm) & $5 \pm 2$ & $9 \pm 2$ & $3 \pm 1$ \\
Length of tertiary level vessels (mm) & $3 \pm 1$ & $2 \pm 0.5$ & $1 \pm 0.5$ \\
\hline
\end{tabular}

Values are presented as the mean \pm mean deviation $(n=8)$. VEGF, vascular endothelial growth factor A; V165, group injected with EMT-6 cells overexpressing VEGF165; V192, group injected with EMT-6 cells overexpressing VEGF192; cV, group injected with EMT-6 cells transfected with control vector pcDNA3.1plus.

was considered to indicate a statistically significant difference between values.

\section{Results}

Overexpression of VEGF165 and VEGF192 in stably transfected EMT-6 cells. EMT-6 cells were stably transfected with either cDNA of VEGF165, VEGF192 or a control vector generating EMT-6 cell clones V165, V192 and cV, respectively, in order to compare their effects on the vascular pattern formation and angiogenic activity in vivo. Nine clones stably overexpressing VEGF192 and eleven clones stably overexpressing VEGF165 were obtained. ELISA was used to screen the $100 \mu \mathrm{g} / \mathrm{ml}$ heparin-treated conditioned media from the G418-resistant clones, which were further confirmed using RT-semi-qPCR (Fig. 1).

Among the VEGF-expressing cells, clones V192-N3 and V165-N6 produced comparable quantities of VEGF (Fig. 1A). These cells were then used in the subsequent angiogenesis and tumorigenesis assays in vivo. V192-N4, which produced a higher level of VEGF192 than V192-N3, was also used to investigate the effects of increased expression of VEGF192 on EMT-6 tumor growth. cV cells were used as a control.
VEGF192 induces a distinct vascular patterning in the skin adjacent to the tumor. EMT-6 clone cells V192-N3, V165-N6 and $\mathrm{cV}$ were each inoculated intradermally to inspect and compare the vascular pattern characteristics and angiogenic activities of VEGF192 and VEGF165 in vivo. When the tumors reached a certain volume $\left(\sim 28 \pm 3 \mathrm{~mm}^{3}\right)$, the mice were sacrificed and the inner surface of their abdominal wall skins, which covered the implant sites, was removed and spread onto filter paper. Images were then captured for analysis. As shown in Fig. 2, the cV-forming tumors were poorly vascularized. The average number of second and third-type vessels surrounding cV-induced tumors were $8 \pm 2$ and $16 \pm 4$, respectively (Table I). However, the tumors formed by V165-N6 and V192-N3 induced a significantly different vascular patterning, as shown in Fig. 2. Highly vascularized tumors were induced by V165-N6, demonstrating a tree-like normal branching; values of second and third-type vessels surrounding V165 were $7 \pm 2$ and $45 \pm 6 \mathrm{~mm}$, respectively (Table I). Regular vascular patterning surrounded the tumor; however, the capillaries spread further around the tumor, with an average secondary vessel length of $9 \pm 2 \mathrm{~mm}$, compared with the corresponding $\mathrm{cV}$ vessels of $5 \pm 2 \mathrm{~mm}$. By contrast, the vascular patterning induced by V192-N3 was dense, irregular and disorganized; capillaries were only observed adjacent to the tumor, with a shorter average secondary vessel length of $3 \pm 1 \mathrm{~mm}$ (Table I).

VEGF192 overexpression slows tumor growth in vivo. The effects of VEGF192 or VEGF165 overexpression on tumor growth were investigated using subcutaneous transplantations of V192-N3, V192-N4, V165-N6 or cV EMT-6 clones into mice. As shown in Fig. 3, each VEGF transfectant demonstrated significant inhibition of tumor growth rates compared to those of the $\mathrm{cV}$ control group from day 13 onwards. Furthermore, from day 19 following inoculation the V165 tumor-forming group demonstrated significantly higher tumor growth rates compared to those of the two V192 groups. In addition, the V192-N4 group appeared to have a slower growth rate compared with that of the V192-N3 group; however, the difference was not statistically significant. Overall the results indicated that the overexpression of VEGF192 correlated with the strong inhibition of tumor growth. 


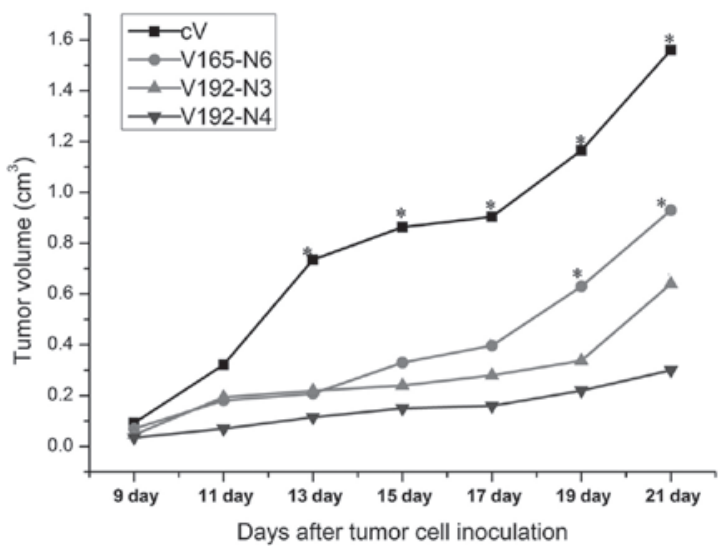

Figure 3. Growth curves of EMT-6-overexpressing VEGF192 and VEGF165 tumors. BALB/c mice were subcutaneously injected with $100 \mu 1$ (1x107 cells/ml RPMI 1640) V192-N3, V192-N4, V165-N6 or cV cells. Tumor diameters were recorded every two days post-injection. Results are expressed as the mean tumor size for each group $(\mathrm{n}=8)$. $\mathrm{P}<0.05$ compared with $\mathrm{cV}$. VEGF, vascular endothelial growth factor A; V165-N6, group injected with EMT-6 cells overexpressing VEGF165; V192-N4 and V192-N3, groups injected with EMT-6 cells overexpressing VEGF192; cV, group injected with EMT-6 cells transfected with control vector pcDNA3.1plus.

VEGF192 overexpression significantly inhibits migration. Wound healing assays were used investigate the effects of VEGF192 or VEGF165 overexpression on the migratory capacity of EMT 6 cells. As shown in Fig. 4A, VEGF192-overexpressing cells showed reduced migration compared to that of the VEGF165-overexpressing and control cells. The number of VEGF192-overexpressing EMT-6 cells migrating into the wound area was significantly decreased compared to that of VEGF165-overexpressing cells and control cells $(\mathrm{P}<0.05)$ (Fig. 4B). This therefore indicated that the overexpression of VEGF192 inhibited EMT-6 cell migration.

Immunohistochemical analysis of microvessel density. As shown in Fig. 5A, there were no obvious areas of necrosis observed in the histological sections from the $\mathrm{cV}$ and V165 cell tumors; however, there was marked necrosis in sections from the V192 cell tumor. In addition, areas of hemorrhage were observed in the V165 and V192 cell tumors.

VEGF165 and VEGF192-transfected clones producing identical quantities of VEGF (V165-N6 and V193-N3) were selected for immunohistochemical analysis of angiogenesis in vivo. Mouse anti-CD31 monoclonal antibodies were used to assess the microvessel density of allograft tumors. The results showed that VEGF165-overexpressing tumors were significantly more vascularized compared with the V192-overexpressing and $\mathrm{cV}$ tumors (Fig. 5B and C). Furthermore, no significant difference was observed between the vascular densities of the VEGF192-overexpressing tumors and those of the $\mathrm{cV}$ tumors; however, the former exhibited a significantly enlarged vascular caliber, with an increased total number of vessels $>10 \mu \mathrm{m}$ in diameter, compared with that of the $\mathrm{cV}$ and V165-overexpressing tumors $(\mathrm{P}<0.05)$ (Fig. 5B and D).

\section{Discussion}

The aim of the present study was to investigate the effect of VEGF192 on angiogenesis and vascular patterning through

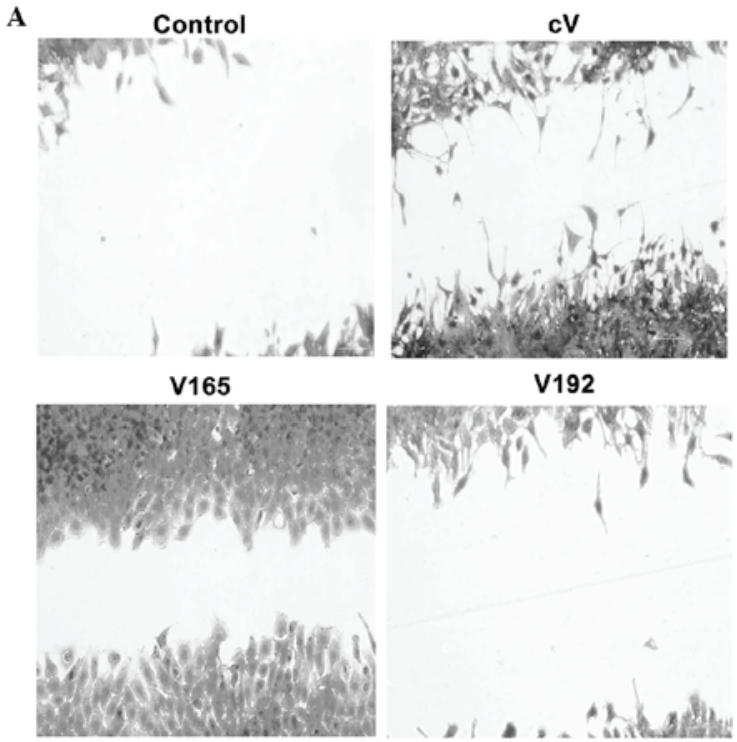

B

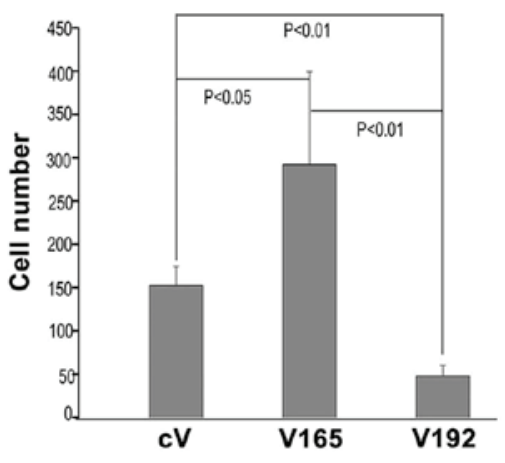

Figure 4. VEGF192 inhibits migration of EMT-6 cells in a wound healing assay. In vitro cell monolayers of EMT-6 cells from each vector group were wounded using the tip of a micropipette and cultured in Dulbecco's modified Eagle's medium for $48 \mathrm{~h}$. (A) Images were captured $48 \mathrm{~h}$ post-injury to detect cell migration. The control indicates the representative size of the wound at the time of injury (magnification, x100). (B) Cell migration was quantified by the number of cells that moved into the wounded area. Data are presented as the mean \pm standard deviation. VEGF, vascular endothelial growth factor A; V165 EMT-6 cells overexpressing VEGF165; V192, EMT-6 cells overexpressing VEGF192; cV, control vector group.

generating EMT-6 murine breast cancer clones which stably overexpressed VEGF192 and VEGF165. Grunstein et al (15) utilized tumor cells which overexpressed an individual isoform of VEGF-A and expressed low levels of endogenous VEGF in order to investigate their effects on tumor angiogenesis. The study reported marked differences in tumor vascularization; however, endogenous VEGF was not eliminated from the invading stroma (15). The results of the present study demonstrated that the overexpression of VEGF192 in EMT-6 tumor cells resulted in increased microvessel density, distinct vascular patterning and a significantly reduced tumor growth rate compared with those of VEGF165-overexpressing tumors.

Previous studies have reported that the vasculature in the adjacent areas to VEGF-overexpressing tumors may also undergo structural alterations $(12,16)$. The VEGF gene produces several isoforms (primarily VEGF121, VEGF165 and VEGF189) through the process of gene splicing. VEGF isoforms commonly differ due to the presence or absence of 

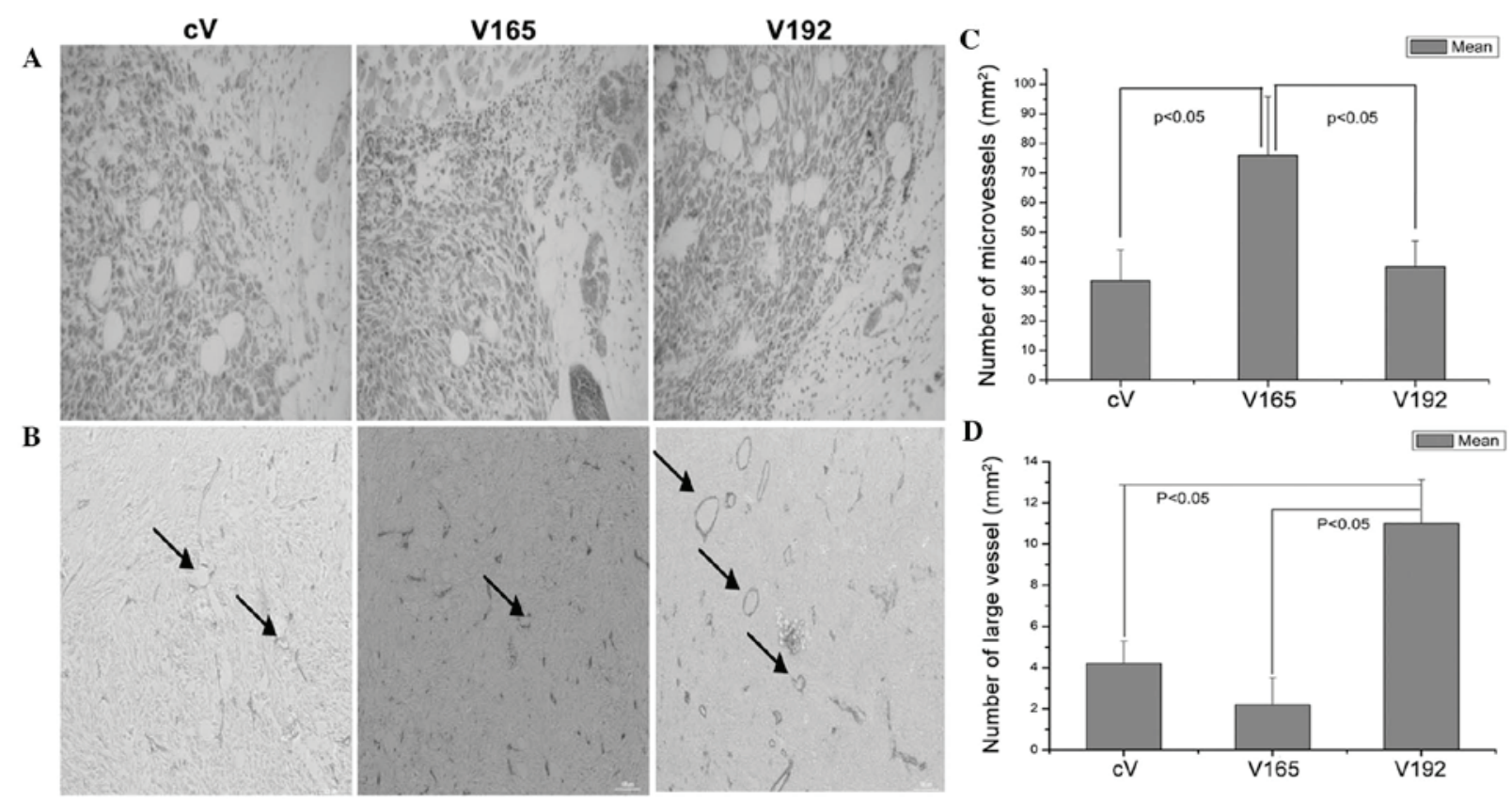

Figure 5. Angiogenesis of the tumors formed by cV, V165 and V192 cells. Tumors were developed in BALB/c mice following intradermal injection of EMT-6-transfected cells. Tumor vascularization was analyzed when tumors reached $28 \pm 3 \mathrm{~mm}^{3}$. (A) Histological analysis of tumors using hematoxylin and eosin staining (magnification, $\mathrm{x} 200$ ). (B) Immunohistochemical analysis of tumor sections labeled with an endothelial cell marker (anti-CD31) (n=6, analysis as performed in triplicate; magnification, x200). Arrows indicate vessels which have a diameter $>10 \mu \mathrm{m}$. (C) Number of microvessels and (D) larger vessels (diameter, $>10 \mu \mathrm{m}$ ) per millimeter squared of tumor tissue was quantified according to the Chalkley method. The vessels were counted in paraffin sections of cV-, V165- and V192-forming tumors $(n=6)$. Data are expressed as the mean \pm standard deviation. VEGF, vascular endothelial growth factor A; V165 EMT-6 cells overexpressing VEGF165; V192, EMT-6 cells overexpressing VEGF192; cV, control vector group.

exons $6 a$ and 7 ; these two exons were reported to determine the diffusible or sequestered (ECM-bound) states of VEGF proteins (17). Numerous studies have confirmed that VEGF isoforms have different roles in vasculogenesis; these studies included evidence from specific VEGF isoform knock-out experiments in mice (18-20). In addition, it was reported that normal VEGF concentration gradients were necessary for regular vascular pattern formation (21); for example, VEGF120/120 mouse embryos, engineered to express a unique isoform of VEGF-A that lacks ECM (heparin)-binding domains, showed significantly decreased capillary branching (18). Conversely, VEGF189 with an ECM-binding domain was reported to provide stimulatory cues that guided endothelial cell-sprouting for the initiation of vascular branch formation (22). The present study hypothesized that VEGF192 may have a higher affinity for ECM compared with that of VEGF165 and therefore may be cleaved by plasmin to release active VEGF isoforms (7). However, it was found that the formed VEGF concentration gradient of VEGF192 was as effective as that of VEGF165. Conversely, VEGF165 was found to have an intermediate affinity for the ECM, with half the VEGF molecules in soluble form, and could therefore form a VEGF concentration gradient. This may therefore explain the difference between the highly-branched, dense and disorganized vascular patterning in tumors of V192-transfected cells and the normal branched, tree-like vascular patterning due to V165-overexpression.

The results of the present study demonstrated that the growth rates of V192-overexpressing tumors were slower than those of V165-overexpressing tumors. A possible explanation for this may be that due to the strong affinity to the ECM V192 was not able to be completely cleaved by MMP or plasmin to form an effective VEGF concentration gradient in vivo, which resulted in a lower vascular density (Fig. 5B). Conversely, the growth rate of specific VEGF isoform-overexpressing tumors in vivo was previously found to be correlated with angiogenic activity as well as the receptor expression profile of the tumor cells (23). Three receptors, namely VEGF receptor (VEGFR) 1, VEGFR2 and neuropilin 1 (NRP1) have been reported to bind VEGF. The expression of these receptors was previously thought to be limited to endothelial cells; however, they have also been reported to be expressed in certain cancer cells $(24,25)$. Furthermore, VEGF has been suggested to be involved in the initiation of cancer cell migration and proliferation (23). Therefore, it may be inferred that the decreased V192 growth rate in the present study was possibly due to the ineffective binding of V192 to NRP1 on EMT-6 cells (NRP1 and VEGFR1 expression were detected in EMT-6 cells using RT-semi-qPCR, data not shown). Hervé et al (26) demonstrated that the interaction of VEGF189 with NRP1 stimulated the proliferation of MDA-MB-231 cells.

The peptide VEGF183 (132-158) contains MMP and plasmin cleavage sites, in addition to the ECM-binding sequence (core sequence, 'KRKRKKSR'). Previous studies have confirmed the interference of the 'KRKRKKSR' sequence with interactions of other heparin-binding growth factors and their receptors (27). In VEGF189, exon 8 is located at the C-terminus; therefore, the 'KRKRKKSR' sequence is not exposed to the C-terminus, preventing direct interference with the VEGF receptors. It is possible that this may be the mechanism by which VEGF189 stimulated the dose-dependent migration of breast cancer cells and proliferation of endothelial cells $(26,28)$. However, VEGF192 was not protected by VEGF exon 8, which may have 
resulted in the interference of the 'KRKRKKSR' sequence with the binding of V192 with the corresponding VEGF receptors. This may therefore explain how VEGF192 overexpression could decrease the growth rate and inhibit the migration of EMT-6 cells.

The microvessel density in tumors of cells transfected with V192 was found to be decreased compared to that of tumors of cells transfected with V165; however, the microvessel caliber in tumors of cells transfected with V192 was significantly increased compared with that of tumors of cells transfected with V165 or $\mathrm{cV}$. This phenomenon could not be explained and further research is required for this to be elucidated. Previous studies have demonstrated that the anchorage of different VEGFs to the ECM may convey differential signaling responses to endothelial cells (29). This therefore indicated that the induced dilation of intratumoral vessels may have resulted from the higher affinity of VEGF189 for the ECM (25).

In conclusion, the results of the present study suggested that one of the functions of the exon 8 in VEGF-A was to prevent the 'KRKRKKSR' sequence from interfering with the interactions between heparin-binding growth factors and their respective receptors. It has therefore been suggested that the fusion of exon 8 to the C-terminus of VEGF192 may be beneficial for its angiogenic activities. In addition, the present study confirmed certain suspected characteristics of VEGF192, including the stronger affinity for the ECM, a longer half-life and release via plasmin-cleavage. However, it is now suggested that VEGF192 may be more beneficial for use in the field of tissue engineering with heparinized materials. Of note, VEGF192 provided a novel insight into VEGF design and indirect evidence for the function of exon 8 of VEGF-A.

\section{Acknowledgements}

This study was supported by grants from the Basic Science and Frontier Technology Planning Project of Henan Province, Educational Commission of Henan Province and Scientific Research Fund of Xinxiang Medical University (nos. 142300410027, 12B320021 and 2013ZD110, respectively).

\section{References}

1. Hoeben A,Landuyt B, Highley MS, Wildiers H, Van Oosterom AT and De Bruijn EA: Vascular endothelial growth factor and angiogenesis. Pharmacol Rev 56: 549-580, 2004.

2. Carmeliet P: Angiogenesis in life, disease and medicine. Nature 438: 932-936, 2005.

3. Lee RJ, Springer ML, Blanco-Bose WE, Shaw R, Ursell PC and Blau HM: VEGF gene delivery to myocardium: deleterious effects of unregulated expression. Circulation 102: 898-901, 2000.

4. Hariawala MD, Horowitz JR, Esakof D, et al: VEGF improves myocardial blood flow but produces EDRF-mediated hypotension in porcine hearts. J Surg Res 63: 77-82, 1996.

5. Simons M: Angiogenesis: where do we stand now? Circulation 111: $1556-1566,2005$

6. Huiyong Z, Yong L, Yunxiao S, Wuling Z, Jingjing L and Taiming L: Generation of a chimeric plasmin-resistant VEGF165/VEGF183 (132-158) protein and its comparative activity. Protein Pept Lett 20: 947-954, 2013.

7. Robinson CJ and Stringer SE: The splice variants of vascular endothelial growth factor (VEGF) and their receptors. J Cell Sci 114 (Pt 5): 853-865, 2001.

8. Tammela T, He Y, Lyytikkä J, et al: Distinct architecture of lymphatic vessels induced by chimeric vascular endothelial growth factor- $\mathrm{C} /$ vascular endothelial growth factor heparin-binding domain fusion proteins. Circ Res 100: 1468-1475, 2007.
9. Simons M: Silky, sticky chimeras-designer VEGFs display their wares. Circ Res 100: 1402-1404, 2007.

10. Keskitalo S, Tammela T, Lyytikkä J, et al: Enhanced capillary formation stimulated by a chimeric vascular endothelial growth factor/vascular endothelial growth factor-C silk domain fusion protein. Circ Res 100: 1460-1467, 2007.

11. Zheng Y, Watanabe M, Kuraishi T, Hattori S, Kai C and Shibuya M: Chimeric VEGF-ENZ7/PIGF specifically binding to VEGFR-2 accelerates skin wound healing via enhancement of neovascularization. Arterioscler Thromb Vasc Biol 27: 503-511, 2007.

12. Lee S, Jilani SM, Nikolova GV, Carpizo D and Iruela-Arispe ML: Processing of VEGF-A by matrix metalloproteinases regulates bioavailability and vascular patterning in tumors. J Cell Biol 169: 681-691, 2005.

13. Lauer G, Sollberg S, Cole M, Krieg T and Eming SA: Generation of a novel proteolysis resistant vascular endothelial growth factor165 variant by a site-directed mutation at the plasmin sensitive cleavage site. FEBS Lett 531: 309-313, 2002.

14. Suhonen KA, Anttila MA, Sillanpää SM, Hämäläinen KM, Saarikoski SV, Juhola M and Kosma VM: Quantification of angiogenesis by the Chalkley method and its prognostic significance in epithelial ovarian cancer. Eur J Cancer 43: 1300-1307, 2007.

15. Grunstein J, Masbad JJ, Hickey R, Giordano F and Johnson RS: Isoforms of vascular endothelial growth factor act in a coordinate fashion to recruit and expand tumor vasculature. Mol Cell Biol 20: 7282-7291, 2000.

16. Mineur P, Colige AC, Deroanne CF, et al: Newly identified biologically active and proteolysis-resistant VEGF-A isoform VEGF111 is induced by genotoxic agents. J Cell Biol 179: 1261-1273, 2007.

17. Ferrara N: Vascular endothelial growth factor. Arterioscler Thromb Vasc Biol 29: 789-791, 2009.

18. Carmeliet P, Ng YS, Nuyens D, et al: Impaired myocardial angiogenesis and ischemic cardiomyopathy in mice lacking the vascular endothelial growth factor isoforms VEGF164 and VEGF188. Nat Med 5: 495-502, 1999.

19. Ruhrberg C, Gerhardt H, Golding M, et al: Spatially restricted patterning cues provided by heparin-binding VEGF-A control blood vessel branching morphogenesis. Genes Dev 16: 2684-2698, 2002.

20. Maes C, Stockmans I, Moermans K, et al: Soluble VEGF isoforms are essential for establishing epiphyseal vascularization and regulating chondrocyte development and survival. J Clin Invest 113: 188-199, 2004.

21. Ferrara N: Binding to the extracellular matrix and proteolytic processing: two key mechanisms regulating vascular endothelial growth factor action. Mol Biol Cell 21: 687-690, 2010.

22. Stalmans I, Ng YS, Rohan R, et al: Arteriolar and venular patterning in retinas of mice selectively expressing VEGF isoforms. J Clin Invest 109: 327-336, 2002.

23. Schoeffner DJ, Matheny SL, Akahane T, et al: VEGF contributes to mammary tumor growth in transgenic mice through paracrine and autocrine mechanisms. Lab Invest 85: 608-623, 2005.

24. Soker S, Takashima S, Miao HQ, Neufeld G and Klagsbrun M: Neuropilin-1 is expressed by endothelial and tumor cells as an isoform-specific receptor for vascular endothelial growth factor. Cell 92: 735-745, 1998.

25. Mercurio AM, Lipscomb EA and Bachelder RE: Non-angiogenic functions of VEGF in breast cancer. J Mammary Gland Biol Neoplasia 10: 283-290, 2005 .

26. Hervé MA, Buteau-Lozano H, Vassy R, et al: Overexpression of vascular endothelial growth factor 189 in breast cancer cells leads to delayed tumor uptake with dilated intratumoral vessels. Am J Pathol 172: 167-178, 2008.

27. Jia H, Jezequel S, Löhr M, et al: Peptides encoded by exon 6 of VEGF inhibit endothelial cell biological responses and angiogenesis induced by VEGF. Biochem Biophys Res Commun 283: 164-173, 2001.

28. Hervé MA, Buteau-Lozano H, Mourah S, Calvo F and Perrot-Applanat M: VEGF189 stimulates endothelial cells proliferation and migration in vitro and up-regulates the expression of Flk-1/KDR mRNA. Exp Cell Res 309: 24-31, 2005.

29. Chen TT, Luque A, Lee S, Anderson SM, Segura T and Iruela-Arispe ML: Anchorage of VEGF to the extracellular matrix conveys differential signaling responses to endothelial cells. J Cell Biol 188: 595-609, 2010. 